\title{
Research on modified selected mapping technique for PAPR reduction and reception performance improvement in 8QAM-OFDM optical access systems
}

\author{
Yufeng Shao* (D) and Wenzhe Ma
}

\begin{abstract}
Background: Orthogonal frequency division multiplexing has been regarded as one of the promising technologies for the optical access systems application. However, high peak-to-average power ratio will cause the signal distortion and enable the used amplifier to operate in nonlinear section.

Methods: In this paper, a modified selected mapping technique is introduced for reducing the peak-to-average power ratio and enhancing reception performance of the downstream 8QAM-OFDM signals in optical access systems.

Results: Compared with the traditional selected mapping technique, the PAPR value of 8QAM-OFDM optical downstream signals with 128 subcarriers is reduced by almost $2 \mathrm{~dB}$ using modified SLM when the complementary cumulative distribution function probability value is 10-2. Moreover, the bit error rate performance will be improved, while 10Gbit/s 8QAM-OFDM optical downlink signals are transmitted over single mode fiber with different access length.

Conclusions: Hence, the proposed scheme becomes more efficient for the future optical orthogonal frequency division multiplexing access systems.
\end{abstract}

Keywords: Optical access system, Orthogonal frequency division multiplexing, Modified selected mapping, Peak-to-average power ratio, Bit error rate

\section{Background}

Orthogonal frequency division multiplexing (OFDM) is the preferred technology in optical access networks since it offer resistance to chromatic dispersion (CD) and polarization mode dispersion (PMD), multiple services access and high spectral efficiency [1, 2]. However, high peak-to-average power ratio (PAPR) not only increases complexity of digital-to-analogue (D/A) and analogueto-digital (A/D) converters, but also causes signals nonlinear distortion since it makes the power amplifier (PA) work in a non-linear area in optical access systems [2-8]. Hence, OFDM signals with low PAPR are desirable for optical signals access. In [9], there are many

\footnotetext{
* Correspondence: syufeng@163.com

Faculty of Engineering, Shanghai Polytechnic University, Shanghai 201209,
} China

PAPR reduction techniques are introduced and analyzed. Amongst these techniques, selected mapping (SLM) is one of the promising methods for reducing PAPR influence because it is simple to achieve and not introduce any distortion [10]. Recently, SLM method is introduced into generation of (16 quadrature amplitude modulation orthogonal frequency division multiplexing) 16QAMOFDM signals in millimeter wave optical access systems for solving high PAPR problem [11, 12]. In Ref. [13, 14], though high PAPR reduction of OFDM using recurring SLM with cyclic block codes scheme and a modified SLM scheme have been proposed, the BER performance has not been analyzed and their applications had not been carried out in communication systems. In this paper, a modified SLM technique is introduced for reducing the PAPR and enhancing reception performance of 
the downstream 8QAM-OFDM signals in optical access systems. Compared with 4QAM-OFDM signals, 8QAMOFDM signals are selected as the downlink signals in this scheme, because 8QAM-OFDM signals can improve system capacity and spectrum efficiency. Moreover, generation and detection of 8QAM-OFDM downstream signals is easier to implement than 16QAM-OFDM signals. The complementary cumulative distribution function (CCDF) curves are measured for analyzing the PAPR influence, when traditional SLM and one modified SLM methods are adopted. Compared with the traditional SLM technique, the PAPR value of 8QAMOFDM optical downstream signals with 128 subcarriers is reduced by almost $2 \mathrm{~dB}$ using modified SLM when the CCDF value is $10^{-2}$. Moreover, the bit error rate (BER) performance will be improved, as 10Gbit/s 8QAMOFDM optical downlink signals are transmitted over single mode fiber (SMF) with different access length using this scheme.

The paper is organized in different sections. Section II gives brief description about system configuration method. Section III discusses the comparison of both proposed method and existing method. Finally, conclusions are given in Section IV.

\section{System configuration}

An OFDM signal can be expressed as

$$
a(t)=\frac{1}{\sqrt{N}} \sum_{n=0^{N-1}} A_{n} e^{j 2 \pi n t / N T}, 0 \leq t \leq N T
$$

where $a(t)$ is an OFDM signal with $N$ subcarriers, and $A_{n}=\left[A_{0,} A_{1}, \cdots A_{(N-1)}\right]$ is an input data sequence. The duration of a data symbol is $T$. The PAPR value of the OFDM signal can be described as

$$
P A P R=\frac{\max \left[\left|A_{n}\right|^{2}\right]}{\mathrm{E}\left[\left|A_{n}\right|^{2}\right]}
$$

Where $\max [$.$] is the maximum value operation, and it$ is indication of maximal value. E[.] is the expectation operator and indication of average power. The above equation represents the ratio of the maximum power value to the average power. In the conventional SLM technique, to reduce the value of PAPR, the input sequence is multiplied by $U$ phase rotation vectors [11]. After Inverse Fast Fourier Transform (IFFT), a few alternative sequences can be obtained (e.g., $X_{u}=\left\{P^{(1)} a, P^{(1)} a, \cdots\right.$, $\left.\left.P^{(N)} a\right\}^{T}, 1 \leq u \leq N\right)$, as shown in Fig. 1a. $X_{k}$ and $X_{m}$ are two of the alternative sequences by using the conventional

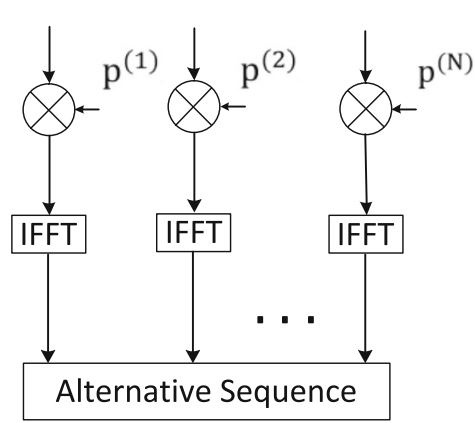

(a)

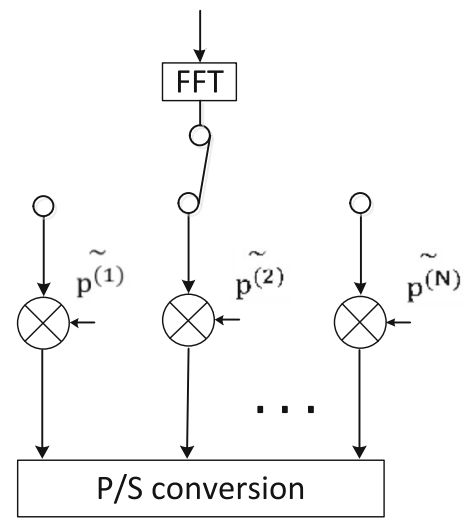

(c)

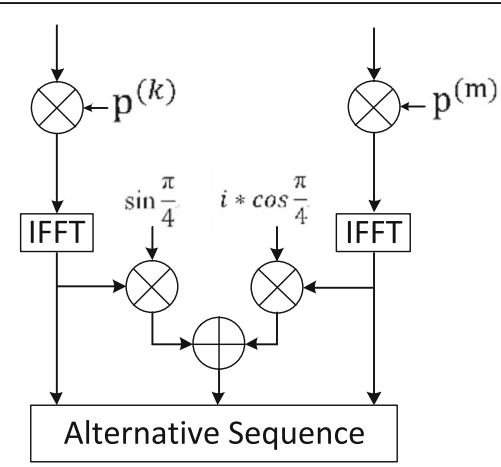

(b)

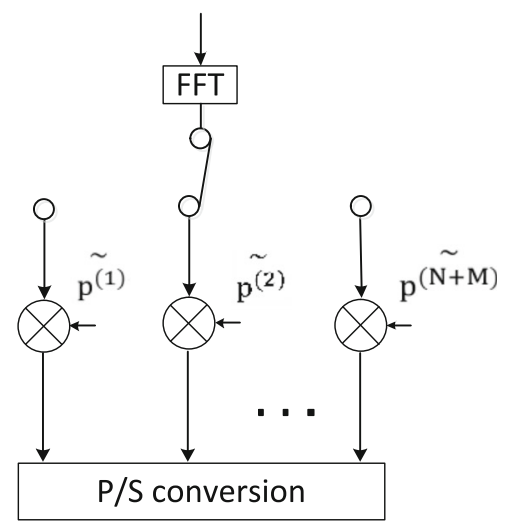

(d)

Fig. 1 a Conventional SLM schematic diagram; b modified SLM schematic diagram; c conventional SLM reverse operation; d modified SLM reverse operation 


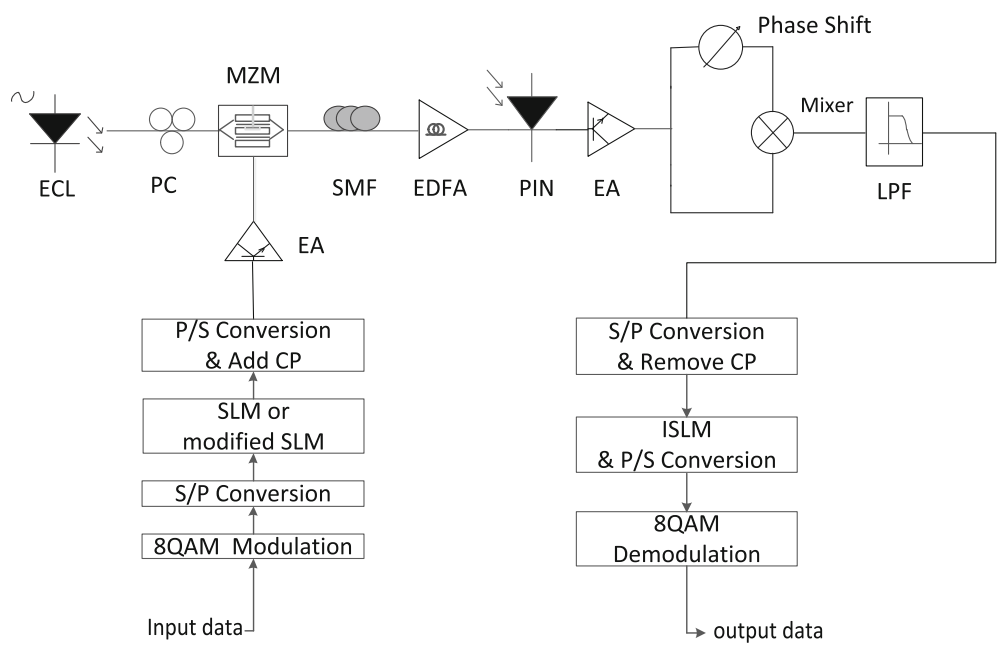

Fig. 2 8QAM-OFDM optical access system. ECL: external cavity laser. PC: polarization controller. MZM: Mach-Zehnder Modulator. EA: electrical amplifier. SMF: single mode fiber. EDFA: erbium doped fiber amplifier. PIN: positive intrinsic-negative. LPF: low pass filter

SLM method. When the modified SLM technique is adopted, the sequences after IFFT are arbitrarily selected out of two. One is multiplied bysin $(\pi / 4)$, and the other is multiplied by $i * \cos (\pi / 4)$, as shown in Fig. 1b. Since Fourier transform has linear property [14], the generated new sequence can be written as

$$
X_{u}=\operatorname{IFFT}\left(a *\left(\sin (\pi / 4) * p^{(k)}+i * \cos (\pi / 4) * p^{(m)}\right)\right)
$$

And then, they are input in the alternative sequence after adding the two together. If extra $M$ alternative sequence is obtained, using this method, $N+M$ alternative sequences will be generated. As shown in Fig. $1 \mathrm{c}$ and d, at the receiver, the OFDM data block can be restored by the reverse operation, which is similar to the used method in Ref. [8]. But, the number of reverse sequence is different.

\section{Methods}

As shown in Fig. 2, at the transmitter, after the 10Gbit/s data are input in 8 QAM modulation module, the generated 8 QAM electric signals are converted from serial signals to parallel signals through one serial-to-parallel converter. And then, the SLM and the modified SLM techniques are used separately for comparing the differences of two methods. In this system, discrete multitone modulation (DMT) and demodulation are also used to generate and receive real value signals, that is similar to the method in Ref. [8]. The parallel-to-serial conversion is adopted before adding cyclic prefix (CP). The generated 8QAM-OFDM electrical signal is input to one Mach-Zehnder modulator (MZM) for completing electro-optic conversion. Simultaneously, one continuous optical carrier is input to the MZM, which is generated by one external cavity laser (ECL) and its line width is $100 \mathrm{kHz}$. The modulator $(\mathrm{V} \pi=3.6 \mathrm{~V})$ is driven by the 8 QAM-OFDM electrical signal with the bias voltage of $1.8 \mathrm{~V}$ for linear modulation. After that, the 8QAM-OFDM optical signal is generated and transmitted over the single mode fiber (SMF, and its parameters are shown in Table 1). Optical spectral diagrams of generated 8QAM-OFDM optical downstream signals using conventional and modified SLM techniques are measured, as shown in Fig. 3. To compensate fiber link attenuation, one adjustable erbium doped fiber amplifier (EDFA) is used. At the receiver, the 8QAM-OFDM optical signal is detected by a high sensitivity positive intrinsic-negative (PIN) and converted to the electrical signal. The electrical signal is amplified by an electric amplifier (EA). And then, the amplified electrical signals are divided into two parts. One is input to one tunable phase shifter for achieving phase adjustment, and the other is transmitted directly. Two parts are input to one electric mixer to achieve self-mixing coherent mixing. Through one low pass filter (LPF) with $3 \mathrm{~dB}$ bandwidth of $7.5 \mathrm{GHz}$, the high frequency noise is filtered out. The 10Gbit/s 8QAM-OFDM electrical signal is converted from one serial signal to one parallel signal by one serial-to-parallel converters. After removing cyclic prefix (CP) and reverse SLM operation, the original input data are got by $8 \mathrm{QAM}$ demodulation.

Table 1 Used optical fiber parameters

\begin{tabular}{ll}
\hline Fiber parameters & SMF \\
\hline Dispersion $(\mathrm{ps} / \mathrm{nm} / \mathrm{km})$ & 16 \\
Fiber loss $(\mathrm{dB} / \mathrm{km})$ & 0.2 \\
Nonlinear refractive index $\left(\mathrm{m}^{2} / \mathrm{W}\right)$ & $2.6 \times 10^{-20}$ \\
Dispersion slope $\left(\mathrm{ps} /\left(\mathrm{nm}^{2} / \mathrm{km}\right)\right)$ & 0.075 \\
\hline
\end{tabular}




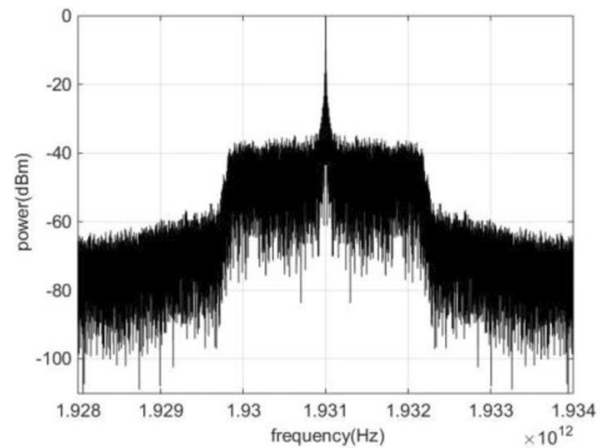

(a)

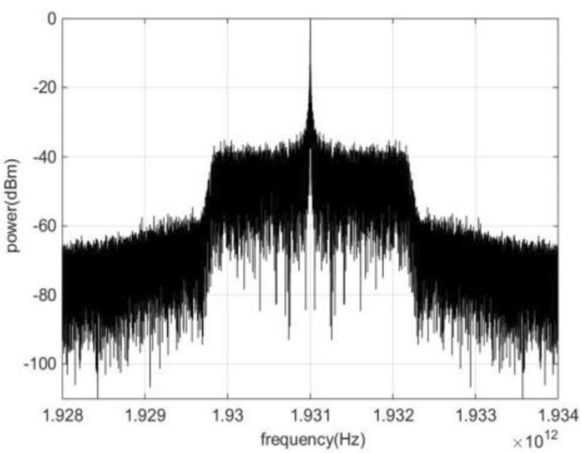

(b)

Fig. 3 Optical spectral diagrams of 8QAM-OFDM optical downstream signals using conventional (a) and modified SLM (b) schemes

\section{Results and discussion}

As shown in Fig. 4a and b, the complementary cumulative distribution function (CCDF) curves of the PAPR using conventional and modified SLM methods, and these cases with different number of subcarriers are compared. While the same number of subcarriers is used, the PAPR probability of $8 \mathrm{QAM}-\mathrm{OFDM}$ signals is lower using the modified SLM method than conventional SLM. Note that, when the number of subcarriers is 128 , the value of PAPR using modified SLM is reduced almost $2 \mathrm{~dB}$ compared with conventional SLM when the CCDF value is $10^{-2}$. No matter what kind of scheme is adopted, with the increase of the number of subcarriers, the value of PAPR will increase. While the number of subcarriers is more, the spectral efficiency of OFDM signals is higher. However, a large number of subcarriers are used will increase the complexity of the DSP and the system cost budget. Hence, 128 is often selected as a tradeoff number of the used subcarriers in optical access system [11]. The BER curves and constellation diagrams for the recovered data using SLM or modified SLM before and after downstream transmission are depicted in Fig. 5. As the downstream transmission distance is $1 \mathrm{~km}$ or less, no matter what kind of scheme is used, the BER values are almost equal and lower than $10^{-9}$. Since in the signal downstream transmission over optical fiber the degradation of signal-to-noise ratio (SNR) becomes larger with the increase of distance, the value of BER will increase and the distribution of constellation points is also more divergent correspondingly. From the constellation diagrams inserted in Fig. 5, at the end of $10 \mathrm{~km}$ and $20 \mathrm{~km}$ optical fiber, the distribution of constellation points is more ideal using modified SLM technique. Hence, as the distance increase, the reception performance of 8QAM-OFDM signals using the modified SLM is better than the conventional SLM. When the transmission distance is $20 \mathrm{~km}$, the BER values of 8QAM-OFDM optical access system using conventional and modified SLM methods are $10^{-3}$ and $10^{-3.5}$ respectively. The results demonstrate using the modified SLM technique can slightly improve the BER perforrmance. As we know, for generating OFDM signals, a variety of forms of QAM are available and some of the more common forms including 4QAM, 8QAM,

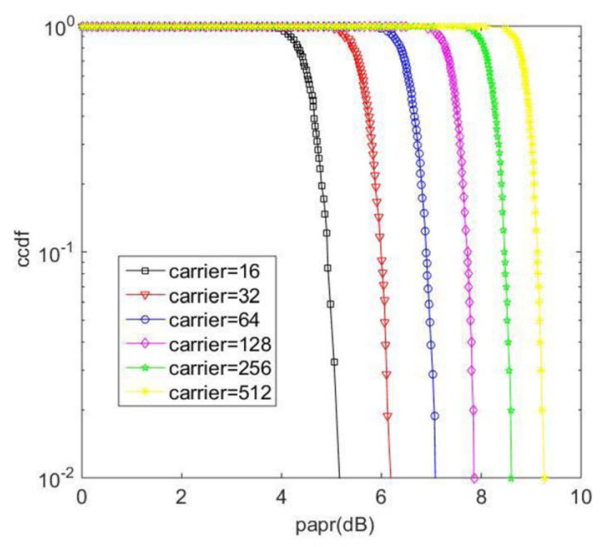

(a)

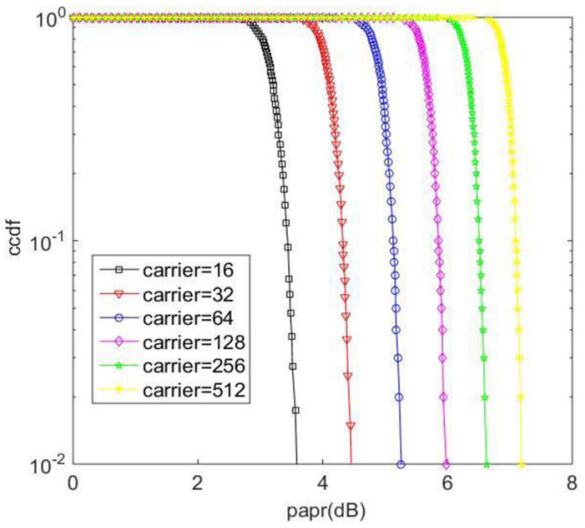

(b)

Fig. 4 CCDF curves of PAPR using conventional (a) and modified SLM (b) schemes 


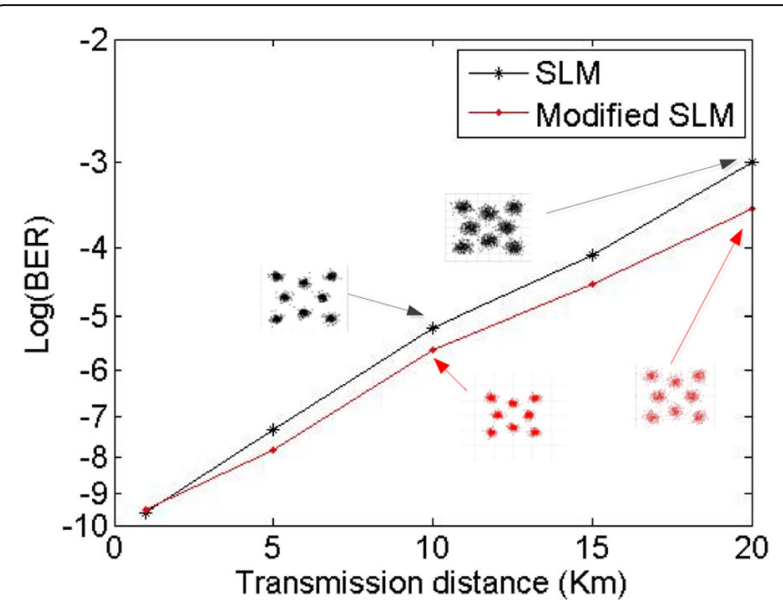

Fig. 5 The relationship between BER and transmission distance

16QAM, 32QAM, 64QAM and 128QAM. Because 4QAM-OFDM, 16QAM-OFDM, 32QAM-OFDM, 64QAM-OFDM and 128QAM-OFDM signals are adopted in the optical access system, the results show that the BER performance is almost the same when using SLM and modified SLM techniques. Moreover, the value of PAPR using modified SLM is not reduced compared with conventional SLM under the same condition, while 4QAM-OFDM, 16QAM-OFDM, 32QAMOFDM, 64QAM-OFDM and 128QAM-OFDM signals are used as the downlink signals. Therefore, this paper only describes 8QAM-OFDM downstream signals application, because using modified SLM technique the values of BER and PAPR are more desirable compared with traditional SLM technique. Hence, the modified SLM technique is a better solution scheme for reducing PAPR influence and enhance the reception performance in the 8QAM-OFDM optical access system.

\section{Conclusions}

For reducing PAPR influence and enhance signal reception performance in the 8QAM-OFDM optical access system, the modified SLM technique is introduced and compared with the traditional SLM method. In this scheme, 8QAM-OFDM optical downstream signals are transmitted successfully over SMF with different access distances at $10 \mathrm{Gbit} / \mathrm{s}$. The results demonstrated that, the PAPR probability of 8 QAM-OFDM signals will be reduced adopting the modified SLM method. Moreover, the reception performance of 8QAM-OFDM signals using the modified SLM is better than the conventional SLM. Hence, the modified SLM method is a potential solution scheme for reducing PAPR influence and enhance the reception performance in future optical access OFDM systems.

\section{Abbreviations}

A/D: Analogue-to-digital; BER: Bit error rate; CCDF: Complementary cumulative distribution function; CD: Chromatic dispersion; CP: Cyclic prefix; D/A: Digital-to-analogue; DMT: Discrete multitone modulation; EA: Electric amplifier; ECL: External cavity laser; EDFA: Erbium doped fiber amplifier; IFFT: Inverse fast Fourier transform; LPF: Low pass filter; MZM: Mach-Zehnder modulator; OFDM: Orthogonal frequency division multiplexing; PA: Power amplifier; PAPR: Peak-to-average power ratio; PC: Polarization controller; PIN: Positive intrinsic-negative; PMD: Polar mode dispersion; QAMOFDM: Quadrature amplitude modulation orthogonal frequency division multiplexing; SLM: Selected mapping; SMF: Single mode fiber; SNR: Signal-tonoise ratio

\section{Acknowledgements}

This work is partially supported by the National Natural Science Foundation of China (No.61107064), Innovation Program of Shanghai Municipal Education Commission (No.15ZZ101), Leading Academic Discipline Project of Information and Communication Engineering of Shanghai Polytechnic University (No.XXKZD1605), School Foundation of Shanghai Polytechnic University (No.EGD14XQD01).

\section{Funding}

This work is partially supported by the National Natural Science Foundation of China (No.61107064), Innovation Program of Shanghai Municipal Education Commission (No.15ZZ101), Leading Academic Discipline Project of Information and Communication Engineering of Shanghai Polytechnic University (No.XXKZD1605), School Foundation of Shanghai Polytechnic University (No.EGD14XQD01).

\section{Availability of data and materials}

All data and materials are fully available without restriction.

\section{Authors' contributions}

YS conceived and designed the study. YS and WMa performed the system configuration, and wrote the manuscript. YS reviewed and edited the paper. Both authors read and approved the manuscript.

\section{Competing interests}

The authors declare that they have no competing interests.

\section{Publisher's Note}

Springer Nature remains neutral with regard to jurisdictional claims in published maps and institutional affiliations.

Received: 31 August 2017 Accepted: 3 November 2017

Published online: 16 November 2017

References

1. Shao, Y., Wang, Y., Chi, N.: 60-GHz RoF system with low PAPR 16QAM-OFDM downlink using PTS segmentation. IEEE Photon. Technol. Lett. 25, 855-858 (2013)

2. Shao, Y., Huang, B., Chi, N., et al.: A novel subcarrier OFDM-MSK WDM passive optical network, p. OTuO4. Optical Fiber Communication Conference, San Diego (2010)

3. Jiang, $T$., Wu, Y.: An overview: peak-to-average power ratio reduction techniques for OFDM signals. IEEE Trans. Broadcast. 54, 257-268 (2008)

4. Wulich, D.: Definition of efficient PAPR in OFDM. IEEE Commun. Lett. 9, 832-834 (2005)

5. Santella, G., Mazzenga, F.: A hybrid analytical-simulation procedure for performance evaluation in M-QAM-OFDM schemes in presence of nonlinear distortions. IEEE Trans. Veh. Technol. 47, 142-151 (1998)

6. Chow, C.W., Yeh, C.H., Xu, L., Tsang, H.: Rayleigh backscattering mitigation using wavelength splitting for heterogeneous optical wired and wireless access. IEEE Photon. Technol. Lett. 22, 1294-1295 (2010)

7. Lin, C.-T., Shih, P.-T., Chen, J., Xue, W., Peng, P., Chi, S.: Optical millimeterwave signal generation using frequency quadrupling technique and no optical filter. IEEE Photon. Technol. Lett. 20, 1027-1029 (2008)

8. Shao, Y.: PAPR reduction and receiver sensitivity improvement in 16QAMOFDM RoF system using DMT modulation and BTN-PS technique. Opt. Laser Technol. 77, 59-63 (2016) 
9. Rahmatallah, Y., Mohan, S.: Peak-to-average power ratio reduction in OFDM systems: a survey and taxonomy. IEEE Commun. Surv. Tutorials. 15, 1567-1592 (2013)

10. Bauml, R.W., Fischer, R.F.H., Huber, J.B.: Reducing the peak-to-average power ratio of multicarrier modulation by selected mapping. Electron. Lett. 32, 2056-2057 (1996)

11. Shao, Y., Chi, N., Fan, J., et al.: Generation of 16-QAM-OFDM signals using selected mapping method and its application in optical millimeter-wave access system. IEEE Photon. Technol. Lett. 24, 1301-1303 (2012)

12. Shao, Y., Ma, W., Hu, Y., et al.: Study on computational complexity in OFDMRoF system using SLM technique, pp. 917-921. Fifth International Conference on Instrumentation \& Measurement, Qinhuangdao (2015)

13. Manhas, P., Soni, M.K.: PAPR reduction of OFDM using recurring SLM with cyclic block codes schemes, pp. 1-5. International Conference on Reliability, Noida (2015)

14. Heo, S.J., Noh, H.S., No, J.S., et al.: A modified SLM scheme with low complexity for PAPR reduction of OFDM systems. IEEE Trans. Broadcast. 53, 804-808 (2008)

\section{Submit your manuscript to a SpringerOpen ${ }^{\circ}$ journal and benefit from:}

- Convenient online submission

- Rigorous peer review

- Open access: articles freely available online

- High visibility within the field

- Retaining the copyright to your article

Submit your next manuscript at $\boldsymbol{s p r i n g e r o p e n . c o m ~}$ 